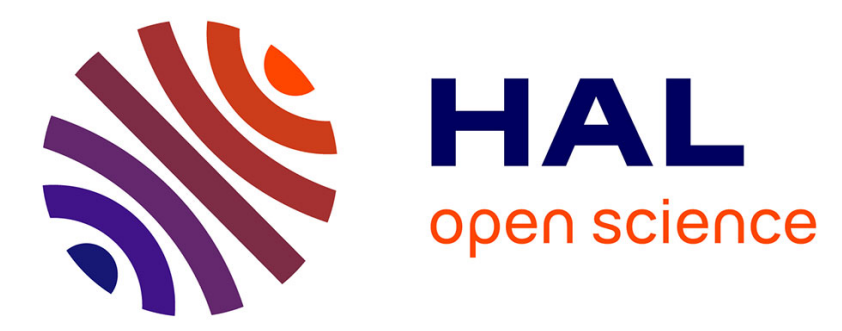

\title{
The role of weak selection and high mutation rates in nearly neutral evolution
}

\author{
Daniel John Lawson, Henrik Jeldtoft Jensen
}

\section{To cite this version:}

Daniel John Lawson, Henrik Jeldtoft Jensen. The role of weak selection and high mutation rates in nearly neutral evolution. Journal of Theoretical Biology, 2009, 257 (4), pp.696. 10.1016/j.jtbi.2008.12.029 . hal-00554557

\section{HAL Id: hal-00554557 \\ https://hal.science/hal-00554557}

Submitted on 11 Jan 2011

HAL is a multi-disciplinary open access archive for the deposit and dissemination of scientific research documents, whether they are published or not. The documents may come from teaching and research institutions in France or abroad, or from public or private research centers.
L'archive ouverte pluridisciplinaire HAL, est destinée au dépôt et à la diffusion de documents scientifiques de niveau recherche, publiés ou non, émanant des établissements d'enseignement et de recherche français ou étrangers, des laboratoires publics ou privés. 


\section{Author's Accepted Manuscript}

The role of weak selection and high mutation rates in nearly neutral evolution

Daniel John Lawson, Henrik Jeldtoft Jensen

PII: S0022-5193(08)00674-7

DOI: doi:10.1016/j.jtbi.2008.12.029

Reference: YJTBI 5417

To appear in: $\quad$ Journal of Theoretical Biology

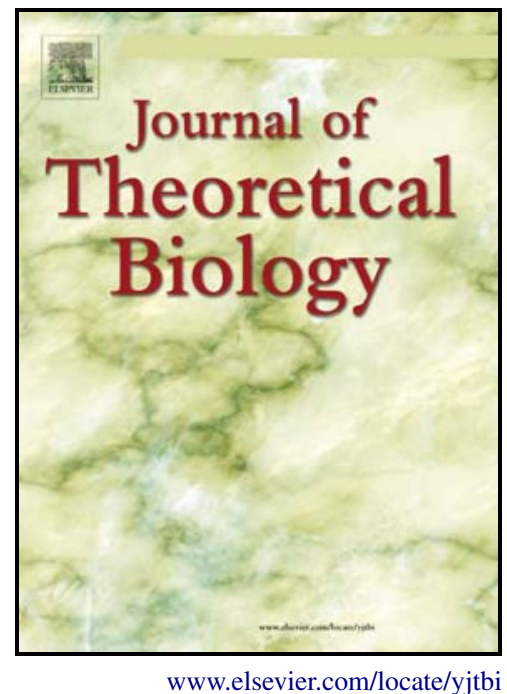

Received date: 1 December 2008

Revised date: 22 December 2008

Accepted date: 23 December 2008

Cite this article as: Daniel John Lawson and Henrik Jeldtoft Jensen, The role of weak selection and high mutation rates in nearly neutral evolution, Journal of Theoretical Biology (2009), doi:10.1016/j.jtbi.2008.12.029

This is a PDF file of an unedited manuscript that has been accepted for publication. As a service to our customers we are providing this early version of the manuscript. The manuscript will undergo copyediting, typesetting, and review of the resulting galley proof before it is published in its final citable form. Please note that during the production process errors may be discovered which could affect the content, and all legal disclaimers that apply to the journal pertain. 


\title{
The role of weak selection and high mutation rates in nearly neutral evolution
}

\author{
Daniel John Lawson* \\ dan.lawson@bristol.ac.uk \\ Biomathematics and Statistics Scotland \\ Macaulay Institute, Craigiebuckler, Aberdeen, UK. AB15 8QH. \\ Present address: Department of Mathematics, University of Bristol, \\ University Walk, Bristol, UK. BS8 1TW. \\ Tel: +44 (0)117928 7990 Fax: +44 (0)117928 7999 \\ Henrik Jeldtoft Jensen \\ h.jensen@imperial.ac.uk \\ Institute for Mathematical Sciences, Imperial College London \\ 53 Princes Gate, South Kensington Campus \\ London, UK. SW7 2PG \\ and: \\ Department of Mathematics, Imperial College London \\ South Kensington campus, London, UK. SW7 2AZ
}

December 22, 2008

\begin{abstract}
Neutral dynamics occur in evolution if all types are 'effectively equal' in their reproductive success, where the definition of 'effectively equal' depends on the population size and the details of mutations. Empirically observed neutral genetic evolution in extremely large clonal populations can only be explained under current models if selection is completely absent. Such models typically consider the case where population dynamics occurs on a different timescale to evolution. However, this assumption is invalid when mutations are not rare in a whole population. We show that
\end{abstract}

${ }^{*}$ Corresponding author. 
this has important consequences for the occurrence of neutral evolution in clonal populations. In highly connected type spaces, neutral dynamics can occur for all population sizes despite significant selective differences, via the forming of effectively neutral networks connecting rare neutral types. Biological implications include an explanation for the high diversity of rare types that survive in large clonal populations, and a theoretical justification for the use of neutral null models.

Keywords: neutral network — fitness landscape — holey landscape

\section{Introduction}

The evolution of a population is influenced by both chance events and selection. Selection acts on a population via differential reproductive success brought about by heritable differences. Chance events include mutations that cause heritable differences and the random process of population dynamics. The perceived relative importance of these various process has changed over time. Darwin [1859] believed that selection with variation was paramount, but more recently Kimura [1983] introduced the 'neutral theory of molecular evolution' demonstrating that chance in population dynamics best describes the fixation of many mutations. This has since been extended to nearly-neutral mutations by Ohta [2003] and others [Tachida, 1991, Nei, 2005]. Very recently the relative importance of chance has again been challenged [Hahn, 2008]. The current genetic inference framework [Felstenstein, 1988] measures phylogenetic relationships in terms of the number of mutations and therefore requires neutral evolution of at least some loci. It is therefore essential to address the relevance of the neutral evolution as a null model.

By current methods it is estimated that up to $50 \%$ of loci in some bacterial genes [Charlesworth and Eyre-Walker, 2006] are shaped by adaptation. This 
leaves a huge proportion of the genome shaped by effectively neutral substitutions. Could undetected selection at these loci be relevant for evolution? $\mathrm{Mu}-$ tations resulting in a small change to reproductive ability are common in both coding and non-coding regions of the genome [Ohta, 1997], arising for example via the stability of RNA folding [Aita et al., 2003], gene regulation [Ohta, 2002] and increased efficiency of shorter genomes. Under the nearly neutral theory of molecular evolution, effectively neutral dynamics are observed for selection less than some critical value which decreases inversely with increasing population size. In a large population of viruses or bacteria selective differences would have to be essentially absent for neutral evolution to occur, but (very) small fitness differences are to be expected in all mutations. We show why neutral evolution is still to be expected for viruses and bacteria.

A standard theoretical approach is to assign 'fitness' to genes under given genetic and environmental conditions, which translates to a reproductive ability for the individual. In sexually reproducing organisms, genes are regularly recombined in different combinations and over evolutionary time an average fitness may be assigned to each gene by averaging over all possible genetic environments. However, in asexually reproducing organisms, recombination is rare and gene interactions are more important in determining long term reproductive success. In this case a better model is to assign a 'fitness' to a combination of genes, i.e. to the type of the individual. Fitness contributions are not independent from each locus. The distinction becomes important when mutations are not rare in a population.

Our model provides an extension to the nearly-neutral theory and permits effectively neutral dynamics for potentially infinite population sizes provided that different genetic types may have the same fitness. An 'effectively neutral' network of types is formed, in which nearest neighbour types need not be com- 
petitively neutral. Competitively neutral types are connected by less fit types in a way that does not affect the statistics of the evolution of the population as a whole. Previous results do not apply in our case because in large populations the usual assumption of the separation of population dynamics from evolutionary dynamics may not hold. The expected number of mutations per generation is large, but the absolute mutation rate need not be high.

Our model predicts the conditions for emergence of neutral networks without a-priori assuming all types are equally viable. Neutral networks have found application to viral evolution [van Nimwegen, 2006] and have been well studied previously [Huynen et al., 1996, van Nimwegen et al., 1999, van Nimwegen and Crutchfield, 2000]. The 'holey fitness landscape' [Gavrilets, 1999] is a theoretically approachable description of neutral networks. Our model is a step towards relating such models with neutral dynamics to non-neutral models.

In Section 2 we define a simple evolution model, for which relevent previous results are interpreted with semi-rigorous arguments in Section 3. Section 4 uses these results to interpret a simulation study before which is discussed in Section 5 .

\section{A conserved population nearly-neutral evolu- tion model}

A simple Moran birth/death process [Moran, 1962] is considered with clonal reproduction in a type space. An individual is characterised by its type, i.e. the alleles at a number of loci. The type determines reproductive probability using a 'fitness landscape' model to describe how reproductive success varies with type. Three possible representative fitness landscapes are considered. 


\subsection{Definition of the model}

A conserved number of individuals $N$ are considered, with each individual $i$ belonging to a given type $\mathbf{x}_{i}=\left(x_{i}^{(1)}, x_{i}^{(2)}, \cdots, x_{i}^{(D)}\right)$ where $D$ is the number of loci and $x_{i}$ is the allele of the $i$ th loci (taking integer values). Each type $\mathbf{x}_{i}$ has a fitness $F\left(\mathbf{x}_{i}\right)$ which determines that types reproduction probability. A generation consists of performing $N$ of the following timesteps:

1. Select an individual $i$ uniformly from the population which will be killed at the end of the timestep.

2. Select an individual $j$ of type $\mathbf{x}_{j}$ for reproduction proportional to its fitness, i.e. with probability $p_{o f f}\left(\mathbf{x}_{j}\right)=F\left(\mathbf{x}_{j}\right) /\left(\sum_{k=1}^{N} F\left(\mathbf{x}_{k}\right)\right)$.

3. Replace individual $i$ with an offspring of individual $j$ with initial type $\mathbf{x}_{i}=\mathbf{x}_{j}$. With probability $u$ a mutation occurs at a single loci, say $\mathbf{x}_{i}^{(\alpha)}$, with $\alpha \in(1, D)$ each chosen with probability $1 / D$. The mutation involves allele $\mathbf{x}_{i}^{(\alpha)}$ changing by +1 or -1 with equal probability.

If all fitnesses $F\left(\mathbf{x}_{j}\right)$ are equal then the dynamics are neutral. We will now define the various fitness landscapes $F(\mathbf{x})$.

\section{$2.2 \quad$ Fitness}

To capture important qualitative features of the change in fitness with type, the following three simple definitions of a fitness landscape are considered.

Landscape 1: The random uncorrelated fitness landscape is maximally rugged, and created by the following function:

$$
F(\mathbf{x} ; s)=1-s y(\mathbf{x})
$$

where $y(\mathbf{x})$ is a random number generated uniformally in $(0,1)$ for each $\mathbf{x}$. Hence 
the fitness $F(\mathbf{x} ; s)$ is uncorrelated between types and is in the range $[1-s, 1]$.

This fitness landscape can also be related to simple correlated (i.e. smoothly varying but random) fitness landscapes by rescaling. Consider a correlated random fitness landscape with correlation length $\eta$, such that $\left\langle F(\mathbf{x}) F\left(\mathbf{x}^{\prime}\right)\right\rangle \propto$ $\exp \left(-\left(\mathbf{x}-\mathbf{x}^{\prime}\right) / \eta\right)$. By rescaling mutation size and mutation rate (i.e. 'coarsegraining' the fitness landscape) the correlated fitness landscape can be reduced to an uncorrelated fitness landscape. Mutation at rate $u$ creates a random walk in type space for a given lineage [Yi-Cheng Zhang et al., 1990] in which the mean population position $\|\mu\|(t)=\left\|\sqrt{\sum_{i=1}^{N} \sum_{d=1}^{D}\left(x_{i}^{d}\right)^{2} / N}\right\| \propto \sqrt{u t}$ at time $t$. Therefore scaling space as $x^{\prime}=x / \eta$ requires scaling mutation rate as $u^{\prime}=$ $u / \eta^{2}$. The correlated fitness landscape in the unprimed variables is described statistically by the random fitness landscape in the primed variables.

Of course, correlations in fitness are important for a range of parameter values [Wilke et al., 2002] and our landscapes consider only the extreme cases. Sufficiently large random correlated landscapes for a finite number of loci require a great amount of care to construct [Laird and Jensen, 2006] and are therefore not considered here. The random landscape is generated by using a pseudorandom number generator with seed given by the location in type space $\mathbf{x}$.

Landscape 2: The truncation fitness landscape is an extreme example of a correlated landscape, given by:

$$
\begin{aligned}
F(\mathbf{x} ; s) & =1, & & \text { if all }\left|\mathbf{x}^{(\alpha)}\right|<L, \\
& =1-s, & & \text { if any }\left|\mathbf{x}^{(\alpha)}\right| \geq L .
\end{aligned}
$$

As before, the label $\alpha \in(1, D)$ refers to directions in type space. Equation 2 describes a 'top hat' function such that fitness decreases by an amount $s$ outside a square (in $D$ dimensions) of side $2 L$. This represents type spaces with a single well defined fit area, and has been well studied in the past [e.g. Kimura and 
Crow, 1978]. As before the maximum fitness gradient is $s$.

Landscape 3: The linear fitness landscape has fitness increasing linearly in all dimensions:

$$
F(\mathbf{x} ; s)=1+s \sum_{\alpha=1}^{D} x^{(\alpha)} .
$$

Again the maximum fitness difference between neighbouring types is $s$, but the maximum fitness difference over the whole population is unbounded (unlike in landscapes 1 and 2). Here each allele contributes additively to fitness, but alternatively multiplicative contributions could be considered. Since such a landscape is more strongly selective than the additive case, it is less relevant to neutral evolution and is therefore not considered here.

\section{Theory}

The nearly-neutral case with high mutation rates is difficult to approach analytically, and so we use simple semi-rigorous but informative 'mean-field' arguments which are backed by numerical simulation. The size of the 'neutral regime' is considered, i.e. the range of selection strengths for which effectively neutral dynamics are observed.

\subsection{Characterisation of neutral dynamics}

Since neutral evolution is itself dynamically rich, a careful characterisation is necessary. To describe a neutrally evolving population statistically we use some theoretical properties of neutral evolution summarised by Lawson and Jensen [2007] without repeating the derivations. A correct model of neutral dynamics is useful for calculations when selection is small but significant, which are usually expanded around the neutral case [e.g. Traulsen et al., 2006].

A neutrally evolving population is described as a statistical distribution with 
two important properties. The average type (or mean position) $\mu(t)$ is a vector of the average alleles for each locus. The standard deviation $w(t)$ describes the variation in the population (which is referred to as a width in the reference). The variables $\mu(t)$ and $w(t)$ are random variables with known behaviours. The average type $\mu(t)$ drifts in time [following a 'random walk', e.g. Bailey, 1964] characterised by:

$$
\langle\|\mu\|\rangle(t) \propto t^{\beta}
$$

with $\beta=1 / 2$ in a euclidean type space, where $\langle\|\mu\|\rangle$ is the expected value of the modulus of the average position. On less connected neutral networks $\beta$ takes different values [de Almeida et al., 2000].

The variation fluctuates strongly around a well defined time-averaged limit $w^{*}$ defined as:

$$
w^{*}=\lim _{T_{m} \rightarrow \infty} \frac{1}{T_{m}} \int_{t=0}^{T_{m}} w(t) d t .
$$

Both the limit $w^{*}$ and its fluctuations can be calculated but for our purposes we only need that the fluctuations are not zero for any population size $N>1$. We measure $\langle w\rangle$, the same quantity as $w^{*}$ without the limit $T_{m} \rightarrow \infty$.

We consider two possible statistically relevant effects of weak selection on the neutral population distribution. The first is a change in $\beta$ : an increase corresponds to directional selection, and a decrease corresponds to stabilising selection towards some fit type. The second effect is a change in $\langle w\rangle$ meaning that selection changes either the variation in the population, or how this fluctuates with time.

To detect a change in variation, we note that the time-averaged variation $w^{*}$ takes a different value to the 'equilibrium' variation $w^{\text {equil }}$, defined to be the value for which the expected change in time is zero:

$$
\left\langle\frac{d w}{d t}\right\rangle_{w=w^{\text {equil }}}=0
$$


This is because there are asymmetric fluctuations in $w$ around $w^{*}$. Selection that acts to keep $w(t)$ more constant will produce a contraction of $\langle w\rangle$ towards $w^{\text {equil }}$, and conversely for selection that increases the variation of $w(t)$. A change in $w^{\text {equil }}$ will likewise produce a change in the time-averaged variation $\langle w\rangle$. Thus $\langle w\rangle$ is an accessible measure characterising neutral dynamics and captures all 'typical' changes to the type distribution (since it characterises several aspects of the distribution at once).

Because $\langle w\rangle$ depends on both the average variation in the population, and the fluctuations in time of the variation, it is a robust indicator of neutral dynamics. Although coalescent methods would have to be used to determine with certainty whether the genealogy was affected by selection, $\langle w\rangle$ will capture 'typical' deviations away from neutrality. For example, a quantity of practical importance is the distribution of genetic distances within a population. For $\langle w\rangle$ to be constant with changing selection despite a changing genetic distance distribution, specific distribution changes must occur which are not typically expected. Numerical simulations (see Appendix A) confirm that $\langle w\rangle$ captures the genetic distance distribution with regard to effectively neutral dynamics.

\section{$3.2 \quad$ Two competing types}

It is instructive to consider the results of 'nearly-neutral theory' reviewed by Ohta [2003], which we recover in our model when mutation rates are low and only two types compete at a given time. The derivation given here differs from the standard one because generations are continuous rather than discrete and our focus is on a haploid population, but the result is the same. The higher mutation rate cases are compared to this simple case.

A mutation occurs in a haploid population of $N$ individuals with fitness $F=1$. The mutant type $i$ is less fit with $F(i)=1-s$. For no selection $(s=0)$, 
the mutant type becomes extinct with probability $1-1 / N$ and so $N$ attempts are needed on average for fixation of the mutant type. The dynamics are called effectively neutral for $s \neq 0$ if a less fit type fixates in $O(N)$ attempts.

The problem is solved under the name of 'Gambler's Ruin' [see e.g. Ash, 1970]. The ratio of the probability of $n_{i}$ increasing to the probability of decreasing is $1-s+O\left(s^{2}\right)$, with $s$ considered small. By comparison to the Gambler's Ruin problem with this ratio, a population of the poor type $i$ of initial size $n_{i}(0)=1$ in a total population of $N$ will eventually reach population size $N$ with probability:

$$
p_{\text {poor }}=\frac{s}{(1+s)^{N}-1} .
$$

The neutral case with $s=0$ succeeds with probability $\hat{p}_{0}=1 / N$. The ratio $p_{\text {poor }} / p_{0}$ is 'exponential like' in $s$ with the characteristic scale:

$$
s^{*}=\frac{2}{N},
$$

or equivalently, effectively neutral evolution is observed for $s<s^{*}=2 / N$. $s^{*}$ is called the critical selection value.

Selection acts in this case by preventing fixation of less fit mutant types, and so 'pinning' the population to a fit type. In this case the above argument can be extended to fitness landscapes with multiple high and low fitness areas. Aranson et al. [1997] perform such an argument mathematically in a slightly different fitness landscape to ours. By analogy to pinning in anomalous diffusion [Bouchaud and Georges, 1990, Ralf Metzler and Joseph Klafter, 2000], if there is some maximum to the time the population can spend at fit sites then a rescaling of the mutation rate will recover standard mutation-drift dynamics. In this case fitness variation is irrelevant over long times. Alternatively, if the time taken to leave fit types is unbounded then the absolute fixation rate of 
mutants decreases with time - this is know as 'subdiffusion'. Mathematically, the average root-mean-square position $\langle\|\mu\|\rangle(t) \propto t^{\beta}$, with $\beta<1 / 2$ and the dynamics are not statistically neutral.

As $u$ increases a range of types can coexist. We will address whether the existence of a population distribution around a high fitness type allows faster transitions between distant fit types, or if the low fitness of the surrounding types prevents the establishment of a wide population distribution.

To summarise the nearly-neutral theory, if mutations are rare $(N u \ll 1)$ and selection is weak $(s \ll 1)$, effectively neutral evolution is expected for $s<s^{*} \propto N^{-1}$.

\subsection{Predictions for a large population in a fitness land- scape}

When mutation rates are high, analytical techniques become difficult and we will resort to simulation. However, some previous results yield predictions which are summarised where available.

Landscape 1: the random uncorrelated fitness landscape as discussed represents a correlated landscape upon rescaling $u$. The critical selection $s^{*} \propto N^{-\alpha}$ is not simple to model theoretically and is the main target of the simulation study.

Landscape 2: the truncation fitness landscape can be understood theoretically as described mathematically by van Nimwegen et al. [1999] for a more general case. The population dynamics between the fit region and the unfit region are related to the two-type case with mutation rate across the fitness boundary depending on the specific population distribution. Within a single region the dynamics are neutral. Since there are effectively only two types competing regardless of $D$, the dynamics follow the low mutation rate case above 
with the upper bound in selection strength for neutral dynamics $s^{*} \propto N^{-1}$.

Landscape 3: the linear fitness landscape can also be understood theoretically at large mutation rates, as discussed in detail by Kessler et al. [1997] for arbitrary selection strength. The variation $w \propto(u N)^{1 / 2}$ for all selection $s$, and therefore the effective fitness difference of individuals within the population is $s_{\text {diff }} \propto$ $s(u N)^{1 / 2}$. The best and worse types compete with small effective mutation rate as in the two type case with $s_{\text {diff }}^{*} \propto N^{-1}$. Therefore $s_{\text {diff }}^{*} \propto s^{*}(u N)^{1 / 2}$ and by rearrangement the upper bound in selection strength for neutral dynamics $s^{*} \propto N^{-3 / 2}$. Additionally, the mean type performs a biased random walk in the usual way [e.g. Bailey, 1964], with deterministic drift component $v \propto s_{\text {diff }} u$ and variance component $\sigma^{2} \propto 1 / N^{2}$. Deterministic drift dominates the random walk if $v>\sigma$, or $s_{\text {diff }}>s_{\text {diff }}^{*} \propto(N u)^{-1}$ and again $s^{*} \propto N^{-3 / 2}$. This holds for arbitrary dimension $D$ as all mutations have an equal chance of increasing or decreasing fitness.

\section{Results}

Since the theoretical predictions for landscapes 2 and 3 correctly describe the simulation results, we focus on the case of landscape 1, the random fitness landscape. Results for all landscapes are presented in summary form. Nonneutral dynamics are observed for $s>s^{*}$, where $s^{*}=\min \left(s_{w}^{*}, s_{\mu}^{*}\right)$ is the critical selection found by observing changes to either the average variation (observed for $s>s_{w}^{*}$ ) or mean type (observed for $s>s_{\mu}^{*}$ ).

We perform ensemble averaging over a large number $(100+)$ of simulations at a range of parameters, and use statistical bootstrapping techniques [Davison and Hinkley, 1997] to provide accurate standard errors. The focus is the relationship between population size and effectively neutral dynamics. To avoid repetition, detailed results are given for the case of low dimension which is well under- 
stood theoretically under truly neutral dynamics [Lawson and Jensen, 2007]. It is important to stress that the general features discussed extend to arbitrary dimension, including the genetically relevant infinite dimension limit.

We use the 'data collapse' method in order to evaluate the functional dependence of the average variation and type on the selection strength $s$ and population size $N$. This works by applying simple transformations to data from several different values of $N$ to obtain the same functional form in $s$; see Appendix B for details.

\subsection{Effects on the average variation}

As discussed, the time-averaged variation in a population provides a strong indicator of neutral dynamics in a population. Fig. 1 (left) shows the average variation in the population as a function of selection $s$. Each population size displays a different 'effectively neutral' region $s<s_{w}^{*}(N, u, D)$ for which selection is invisible and the variation $w(s)=w(0)$. As selection is increased above $s_{w}^{*}$ the time-average variation decreases, either as a result in a change to the equilibrium variation or the nature of fluctuations in the variation.

Considering only the effect of $N$ on $s_{w}^{*}$, the neutral data can be collapsed as $s^{\prime}=s / s_{w}^{*}(N)$ and $w^{\prime}(s)=w(s) / w(s=0)$ shown in Fig. 1 (right), with $s_{w}^{*}$ shown as a vertical line. Accurate confidence intervals for $s_{w}^{*}$ are found by statistical bootstrapping as described in Appendix B. For $D=1$ the critical selection is $s^{*}(N)=8 N^{-0.94}$ (see Fig. 2 for error margins). The data collapse is intended only for $s<s^{*}$, though in this case holds over the whole parameter region. The region $s>s^{*}$ corresponds to non-neutral dynamics. The critical selection observed via a change in the average variation is of the general form:

$$
s_{w}^{*}=a N^{-\alpha(D, u)} D^{\beta(u)},
$$


where $a$ is a constant, $\beta$ is an exponent for the dimension dependence and $\alpha$ for population size dependence. We focus on the case of constant $u$ such that $s_{w}^{*} \propto N^{-\alpha(D)}$ and measure $\alpha(D)$.

The data for landscape 2 (the top-hat fitness landscape) is fit similarly to the Random Landscape. For landscape 3 (the linear fitness landscape), the fitting procedure is more difficult since a general nonlinear form must be used for $w(s)$, so averages and plausible ranges of $s^{*}$ are estimated by eye for this case only.

The collapsed data for all cases and dimensions resemble Fig. 1 (right) with only $\alpha$ differing. The functional form $\alpha(D)$ is summarised in Fig. 2. Theoretical predictions for the scaling of the critical selection $s^{*} \propto N^{-\alpha(D)}$ are supported: for landscape 2: the truncation landscape $\alpha(D)=1$, and for landscape 3: the linear landscape $\alpha(D)=3 / 2$. For landscape 1: the random fitness landscape $\alpha(D)$ has a clear relationship with dimension, starting in $D=1$ at a value away from $\alpha=1$ and decreasing (close to linearly) with dimension towards $\alpha=0$ where it remains for $D \rightarrow \infty$. The gradient $\alpha(D)$ (for $\alpha>0$ ) is mutation-rate dependent.

\subsection{Changes in the average type}

In the neutral case, the average type is a random variable performing a 'random walk', that is, it undergoes drift according to $\langle\|\mu\|\rangle(t) \propto t^{\beta}$ with $\beta=1 / 2$. When selection leads to fixation of a high fitness type then $\beta<1 / 2$, and in a fitness gradient $\beta>1 / 2$. Using Eq. 4 a simplistic method to find the diffusion exponent $\beta$ is as the regression coefficient when plotting $\|\mu\|$ as a function of time $t$ on double logarithmic axes.

The evolution of a population in a random fitness landscape is related to the behaviour of a random walker in a random potential. Studying this numerically is notoriously difficult [Bouchaud and Georges, 1990] and the above method 
poorly captures the asymptotic behaviour. However, the short and medium time scales that are of relevance to biological evolution are captured in $\beta$ as measured by the above regression method.

As expected from Eq. 4, $\beta=1 / 2$ is observed for neutral dynamics as seen for all selection strengths $s<s_{\mu}^{*}$ (not shown). The critical selection observed $s_{\mu}^{*}$ in the mean position has the form

$$
s_{\mu}^{*}=b D^{\gamma}(u)
$$

for some constant $b$ and exponent $\gamma$, with no dependence on population size $N$ for all $N \geq 500$.

\subsection{Variation as the important measure}

Using the definition of $s^{*}=\min \left(s_{w}^{*}, s_{\mu}^{*}\right)$ and Equations 9 and 10, the dynamics of the mean type provide an important constraint on neutral dynamics if $s_{\mu}^{*}<s_{w}^{*}$. By rearrangement:

$$
\alpha \log (N)+(\gamma-\beta) \log (D)<[\log (a)-\log (b)]
$$

Therefore the signs of $(\gamma-\beta)$ and $[\log (a)-\log (b)]$ determine which constraint holds as $N$ and $D$ become large. By regression in the dimension variable $D$, these are empirically observed as positive over all tested parameter values. For example, at $u=0.05$ in the random landscape $(\gamma-\beta)=4.5 \pm 0.7$ and $[\log (a)-$ $\log (b)]=6.9 \pm 0.8$. The signs of the combined constants imply all terms in Equation 11 are positive. Therefore at low $N$ and $D$ a change in the mutational drift rate can be observed before the population distribution changes shape. However, if $N \rightarrow \infty$ or $D \rightarrow \infty$ then $s_{w}^{*}<s_{\mu}^{*}$ and $s^{*}=s_{w}^{*}$, i.e. we need only observe the time-averaged variation. 


\subsection{Interpreting the results}

A statistical description of neutral evolution was used to characterise the effect of selection on a population distribution. This defined a 'neutral regime' in which the population as a whole evolved effectively neutrally.

For low mutation rates $N u \ll 1$ all individuals are distributed over a maximum of two types that compete with each other. In this case neutral dynamics are observed for $s<s^{*} \propto N^{-\alpha}$ with $\alpha=1$, as is found in classical models. This occurs regardless of the distribution of fitter types in the fitness landscape.

For high mutation rates $N u \geq 1$ the population forms a distribution over many types. The relevant selection parameter $s$ measures the maximum range of fitnesses experienced by the population. Fitness landscapes with a single maxima (Eq. 2), or with long range trends (Eq. 3 with $s$ redefined as $s_{\text {diff }}$ ) also have critical selection $s^{*} \propto N^{-\alpha}$ with $\alpha=1$. However, fitness landscapes with large fluctuations but no long distance trend (Eq. 1) allow neutral dynamics to be observed for a larger range of population sizes $N$. In suitably connected fitness landscapes such as that of genotypes $(D \rightarrow \infty)$ there is no effect of population size on the critical selection strength $s^{*}$. When selection is below $s^{*}$, taking the limit $N \rightarrow \infty$ results in a neutral model. The uncorrelated random fitness landscape is important because correlated random landscapes tend (as $1 / \sqrt{N})$ to uncorrelated ones as $N \rightarrow \infty$.

In the neutral regime for high mutation rates, the population contains a large number of types with differing fitness. The fitness difference may be large enough to be measured as selectively important at the level of single mutations, but is ignored at the population distribution level. Fig. 3 illustrates how distinct fit neutral types may be connected by less fit types. Neutral types do not have to be adjacent, but only within fluctuation range of each other, which is of extension $\sqrt{u N}$ mutations. It is only when selection strength $s>s^{*}$ (of order 
1) that crossing unfit regions becomes unlikely.

Fig. 3 illustrates the importance of connectivity and hence the dimensional dependence for neutral dynamics to be observed. Percolation theory [Grimmett, 1999] has been used to describe evolution on holey fitness landscapes [Gravner et al., 2007], but it is difficult to apply to our case because the population forms a wide distribution. As $N \rightarrow \infty$ the proportion of truly neutral types tends to zero and the fluctuation region becomes infinite. The fluctuation region of a fit type contains intermediate fitness types (grey in Fig. 3) for which the extinction is deterministically sure but occurs slowly. There is no well determined threshold for these types as it depends on the distance from a fit type. Hence the percolation argument as presented here should not be taken beyond an illustrative 'cartoon', although the simple linear relationship of $\alpha$ with dimension in Fig. 2 is indication that an analytical approach may be fruitful. Using an alternative argument, van Nimwegen and Crutchfield [2000] evaluate the time taken to cross variable sized fitness barriers, but the argument does not extend easily to the case of a random fitness landscape.

\section{Discussion}

\subsection{Relation to other works}

We have described a mechanism by which a neutral network may form for large clonal populations when fitness variation can occur on every mutation. This provides theoretical support to models representing large populations with neutral networks [van Nimwegen, 2006] and holey fitness landscapes [Gavrilets, 1999, Drossel, 2001]. The model shows that a neutral network can form without a-priori assuming that all types are equally viable.

Alternative models have been proposed that observe effectively neutral dy- 
namics under selective conditions. Gillespie [2001] examines strong selection and recombination, and finds that strong linkage of a neutral locus to a highly selected locus permits neutral dynamics at the neutral locus at a rate independent of population size. Lynch [2006] discusses how a similar effect is produced by Eucaryotic genetic structure. Our result is the first that explains the preponderance of apparently neutral selection for the large populations of effectively clonal, prokaryotic organisms such as Bacteria.

The underlying mechanism for neutral dynamics occurring in non-equal types is the formation of an effectively neutral network of types via a rugged fitness landscape produced by gene interactions. The NK model for gene interactions [Kauffman and Levin, 1987] provides one theoretical framework in which very rugged fitness landscapes can occur in sexually reproducing organisms, which has been studied [Ohta, 1997] in the nearly neutral model of molecular evolution for low mutation rates.

An opposing effect in which neutral dynamics may lead to observed selection can be seen for evolution on a neutral network [van Nimwegen et al., 1999, Wilke, 2001, Forster et al., 2006]. This selection for mutational robustness leads to populations spending the majority of their evolutionary time away from unfit areas. Two differences may explain the apparent contradiction: firstly, our model does not consider loci for which a large loss of fitness can occur on a mutation. Secondly, we consider 'fitness' to be an unobservable modelling tool, and therefore detect neutrality via changes to mutational drift rather than considering the average fitness in a population. A deeper understanding of the relation between these models is needed.

In many other theoretical studies a large population $N \rightarrow \infty$ is assumed. This limit is not consistent with the assumption of small mutation $N u \ll 1$ and therefore a distribution of types must be considered. Under weak selec- 
tion, we found stochastic evolution models are more typically appropriate to the high dimensional space of genotypes (justifying the Coalescent [Donnelly and Tavare, 1995]) or perhaps complex ecological traits (relating to Ecological Neutral Theory [Hubbell, 2001]). Deterministic evolution models such as Adaptive Dynamics [Waxman and Gavrilets, 2005] are more appropriate to strong or directed selection and simple phenotypic traits. Our model therefore provides important links between previously unrelated models, and provides an explanation of the large number of cryptic asexual species.

\subsection{Biological implications}

The reproductive ability of real organisms is not well described by a static fitness landscape over evolutionary time. Reproductive success is caused by a wide range of features including environmental effects and interaction of individuals. However, each of the landscapes discussed may qualitatively describe individuals in a population for a time. The linear fitness landscape describes directional selection, the truncation fitness landscape describes stabilising selection, and the random fitness landscape is appropriate when there is fitness variation without long range trends. We would therefore expect to observe each behaviour only in a subset of type space for a given evolutionary environment.

The most important contribution from our model is a theoretical justification for the widespread use of stochastic models of genetic evolution for large populations. Neutral or nearly neutral evolution can be a reasonable assumption for large clonally reproducing populations even when there is fitness variation between types. It is important to integrate the effects of large mutation rates and high population sizes into the current theoretical frameworks. Although population size cannot be inferred from genetics data alone [Stephens, 2007] our model demonstrates that the qualitative nature of dynamics need not change 
with population size. Hence stochastic models for changing population sizes experiencing weak selection are also reasonable. Our model is not directly applicable to genetics data, but does translate conceptually to problems involving mutation of DNA. Neutral dynamics may naturally occur under different selective conditions for recombining and non-recombining areas, which may be important to inference about mitochondrial DNA [William et al., 1995, Rand et al., 1994] and the Y chromosome (e.g. [Handley et al., 2006]).

The most useful model of biological evolution will differ from situation to situation, particularly depending on the speed of recombination. Our model best describes low recombination rates and therefore asexual populations. It predicts that neutral dynamics can persist for much larger selection strength and population sizes than standard models for sexual species predict. This explains the existence of cryptic asexual species. The conditions required for our model are that mutation rate is not small compared to the inverse population size, and that genes interact to determine fitness. In this case a surprisingly 'large' selective advantage may be present in a population and neutral dynamics can still be observed.

\section{A Genetic distance within the population}

We compare the measurement of $\langle w\rangle$ to the measurement of the time-average population genetic distance $\langle d\rangle$ and a measure of its variance $\left\langle d^{2}\right\rangle$. Fig. 4 illustrates a typical case for $N=100$ and $D=3$. All three measures show the same critical selection strength $s^{*}=0.16$ and the average variation and genetic distance show the same variability. The measure $\left\langle d^{2}\right\rangle$ is more variable because it is a higher moment, but displays the same value of $s^{*}$.

Comparisons over multiple $D \in(1,5)$ and $N \in(100,1000)$ show the same behaviour, with a correlation of $>0.99$ between $\langle w\rangle(s)$ and $\langle d\rangle(s)$ and same 
observed $s^{*}$ in all cases (not shown). Higher values of $N$ have not been tested as calculating $\langle d\rangle$ is computationally demanding and sampling from $\langle d\rangle$ reduces accuracy.

\section{B Data collapse}

The data are collapsed to a single curve for $s<s^{*}$ by normalising the variation and selection. The variation is normalised to 1 at zero selection, for which truly neutral dynamics are observed. Selection is normalised to $s^{\prime}=s / N^{\alpha}$, where $\alpha$ is a dimension dependent constant to be determined.

To determine $\alpha$ we fit a broken-stick model to the data, i.e. a piecewise linear function with two pieces. This is defined by $\log (w)=c_{1}$ for $s<s^{*}$, and $\log (w)=c_{2}+c_{3} \log (s)$ for $s>s^{*}$. This is fit by maximum likelihood of the model parameters given the datapoints.

To determine confidence intervals for $s^{*}$ and $\alpha$ we use a 'bootstrap' method. Since observations were taken at specific values of selection $s_{i}$ there is uncertainty in the values of $w$ for the $s$ in between. Sample $s_{i}$ are obtained by perturbing each $s_{i}$ by an amount $x_{i}$, with a 'tent' distribution of mean $s_{i}$ and extension $\left(s_{i+1}-s_{i-1}\right) / 4$ (i.e. the mean halfway point to its neighbouring points). Sample variations $w_{i}$ for each value of selection $s_{i}$ are obtained via a bootstrapping of the $n$ runs (that is, the average of resampling $n$ values with replacement). This provides a distribution of $w_{i}$ and $s_{i}$. These are once again bootstrapped, so that each $w_{i}$ is sampled and a maximum likelihood $s^{*}$ is obtained, giving a distribution for $s^{*}$. A linear regression is obtained for $\log \left(s^{*}\right)$ as a function of $\log (N)$, giving $-\alpha$ as the slope. Confidence intervals are obtained via the regression variance. 


\section{Acknowledgements}

The work was partially funded by the Scottish Government's Rural and Environment Research and Analysis Directorate (RERAD), and the Engineering and Physical Sciences Research Council (EPSRC). We are grateful to Claus Wilke for constructive comments.

\section{References}

Takuyo Aita, Motonori Ota, and Yuzuru Husimi. An in silico exploration of the neutral network in protein sequence space. J. Theor. Biol., 221:599-613, 2003.

Igor Aranson, Lev Tsimring, and Valerii Vinokur. Evolution on a rugged landscape: Pinning and aging. Phys. Rev. Lett., 79:3298-3301, 1997.

Robert B. Ash. Basic Probability Theory. John Wiley \& Sons, Inc., London, 1970.

Norman T. J. Bailey. The elements of Stochastic Processes. John Wiley \& Sons, Inc, New York, 1964.

Jean-Philippe Bouchaud and Antoine Georges. Anomalous diffusion in disordered media: Statistical mechanisms, models and physical applications. Phys. Rep., 195:127-293, 1990.

Jane Charlesworth and Adam Eyre-Walker. The rate of adaptive evolution in enteric bacteria. Mol. Biol. and Evol., 23:1348-1356, 2006.

Charles Darwin. The Origin of Species. Penguin, Penguin Books Ltd, 27 Wrights Lane, London W8 5TZ England, 1859. 
A. C. Davison and D. Hinkley. Bootstrap Methods and their Applications. Cambridge University Press, 1997.

R.M.C. de Almeida, N. Lemke, and I.A. Campbell. Stretched exponential relaxation on the hypercube and the glass transition. Eur. Phys. J. B, 18:513-518, 2000.

Peter Donnelly and Simon Tavare. Coalescents and genealogical structure under neutrality. Annu. Rev. Genet., 29:401-421, 1995.

Barbara Drossel. Biological evolution and statistical physics. Advances in Physics, 50(2):209-295, 2001.

J. Felstenstein. Phylogenies from molecular sequences: inference and reliability. Ann. Rev. Genetics, 22:521-565, 1988.

Robert Forster, Christoph Adami, and Claus O. Wilke. Selection for mutational robustness in finite populations. J. Theor. Biol., 243:181-190, 2006.

Sergey Gavrilets. A dynamical theory of speciation on holey adaptive landscapes. American Naturalist, 154, 1999.

John H. Gillespie. Is the population size of a species relevant to its evolution? Evolution, 55:2161-2169, 2001.

Janko Gravner, Damien Pitman, and Sergey Gavrilets. Percolation on tness landscapes: Effects of correlation, phenotype, and incompatibilities. J. Theor. Biol., 248:627-645, 2007.

Geoffrey Grimmett. Percolation. Springer, 1999. Second Edition.

Matthew W. Hahn. Toward a selection theory of molecular evolution. Evolution, 62:255-265, 2008. 
Lori J. Lawson Handley, Laura Berset-Brőndli, and Nicolas Perrin. Disentangling reasons for low y chromosome variation in the greater white-toothed shrew (crocidura russula). Genetics, 173:935-942, 2006.

Stephen Hubbell. The Unified Neutral Theory of Biodiversity and Biogeography. Princeton University Press, 41 William Street, Princeton, New Jersey 08540, 2001.

M. Huynen, P. Stadler, and W. Fontana. Smoothness within ruggedness: The role of neutrality in adaptation. Proc. Natl. Acad. Sci., 93:397-401, 1996.

S. Kauffman and S. Levin. Towards a general theory of adaptive walks on rugged landscapes. J. Theor. Biol., 128:11-45, 1987.

David A. Kessler, Herbert Levine, Douglas Ridgway, and Lev Tsimring. Evolution of a smooth landscape. J. Stat. Phys., 87:519-544, 1997.

Motoo Kimura. The neutral theory of molecular evolution. Cambridge University Press, The Pitt Building, Trumpington Street, Cambridge CB2 1RP, 1983.

Motoo Kimura and James F. Crow. Effect of overall phenotypic selection on genetic change at individual loci. Genetics, 75:6168-6171, 1978.

Simon Laird and Henrik Jeldtoft Jensen. The tangled nature model with inheritance and constraint: Evolutionary ecology constricted by a conserved resource. Ecological Complexity, 3:253-262, 2006.

Daniel John Lawson and Henrik Jeldtoft Jensen. Neutral evolution in a biological population as diffusion in phenotype space: Reproduction with local mutation but without selection. Phys. Rev. Lett., 98:098102, 2007.

Michael Lynch. The origins of eukaryotic gene structure. Mol. Biol. Evol., 23: 450-468, 2006. 
P. A. P. Moran. The Statistical Processes of Evolutionary Theory. Clarendon Press, Oxford, 1962.

Masatoshi Nei. Selectionism and neutralism in molecular evolution. Mol. Biol. and Evol., 22:2318-2342, 2005.

Tomoko Ohta. The meaning of near-neutrality at coding and non-coding regions. Gene, 205:261-267, 1997.

Tomoko Ohta. Near-neutrality in evolution of genes and gene regulation. Proc. Nat. Acad. Sci., 99:16134-16137, 2002.

Tomoko Ohta. Origin of the neutral and nearly neutral theories of evolution. $J$. Biosci., 28:371-377, 2003.

Ralf Metzler and Joseph Klafter. The random walk's guide to anomalous diffusion: a fractional dynamics approach. Phys. Rep., 339:1-77, 2000.

D. M. Rand, M. Dorfsman, and L. M. Kann. Neutral and non-neutral evolution of drosophila mitochondrial dna. Genetics, 138:741-756, 1994.

M. Stephens. Inference under the coalescent. In D. J. Balding, M. Bishop, and C. Cannings, editors, Handbook of Statistical Genetics, 3rd Ed. John Wiley \& Sons, Inc., 2007.

Hidenori Tachida. A Study on a Nearly Neutral Model in Finite Populations. Genetics, 128:183-192, 1991.

Arne Traulsen, Jens Christian Claussen, and Christoph Hauert. Coevolutionary dynamics in large, but finite populations. Phys. Rev. E, 74:011901, 2006.

Erik van Nimwegen. Influenza escapes immunity along neutral networks. Science, pages 1884-1886, 2006. 
Erik van Nimwegen and James P. Crutchfield. Metastable evolutionary dynamics: Crossing fitness barriers or escaping via neutral paths? Bul. Math. Biol., 62:799-848, 2000.

Erik van Nimwegen, James P. Crutchfield, and Martijn Huynen. Neutral evolution of mutational robustness. Proc. Nat. Acad. Sci., 96:9716-9720, 1999.

D. Waxman and S. Gavrilets. 20 questions on adaptive dynamics. J. Evol. Biol., 18:1139-1154, 2005.

Claus O. Wilke. Adaptive evolution on neutral networks. Bul. Math. Biol., 63: $715730,2001$.

Claus O. Wilke, Paulo R. A. Campos, and José F. Fontanari. Genealogical process on a correlated fitness landscape. J. Exp. Zool. (Mol. Dev. Evol.), 294:274284, 2002.

J. William, O. Ballard, and Martin Kreitman. Is mitochondrial dna a strictly neutral marker? Trends in Ecology and Evolution, 10:485-488, 1995.

Yi-Cheng Zhang, Maurizio Serva, and Mikhail Polikarpov. Diffusion Reproduction Processes. J. Stat. Phys., 58:849-861, 1990. 

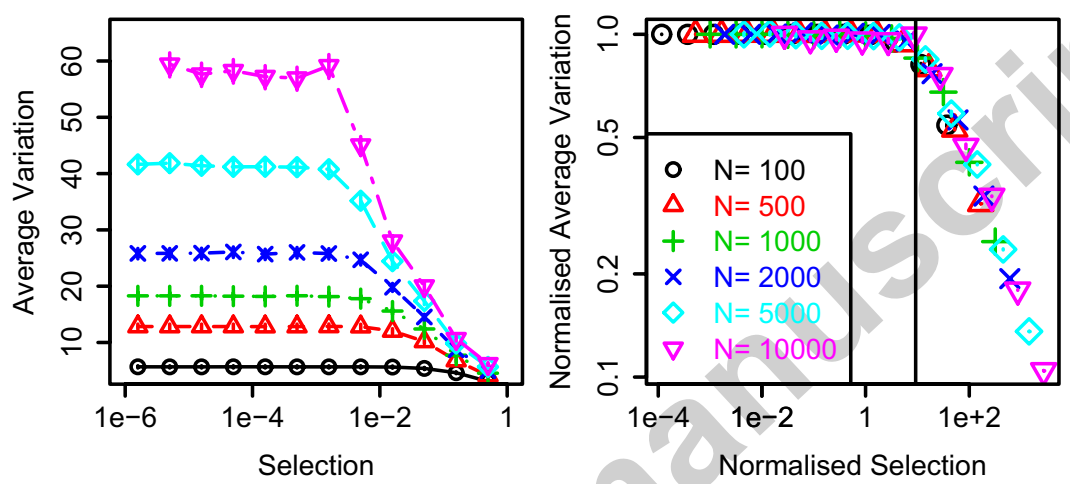

Figure 1: Ensemble averaged variation against selection for fitness landscape 1 (random and uncorrelated) as described by Eq. 1 with mutation probability $u=0.5$ and dimension $D=1$, for a range of population sizes $N$. Left: Ensemble averaged variation against selection. Each curve is flat for $s<s_{w}^{*}(N)$ i.e. $w(s<$ $\left.s_{w}^{*}\right)=w(0)$. Right: The normalised variation against normalised selection for the same data, collapsed using the method from the Appendix. The critical selection $s_{w}^{*} \propto N^{-0.94}$ is shown as a vertical line as an aid to the eye. Each datapoint is the time average (over 50000 generations) of a simulation after it has reached equilibrium, ensemble averaged over 200 independent runs with standard deviations calculated using statistical bootstrapping. 


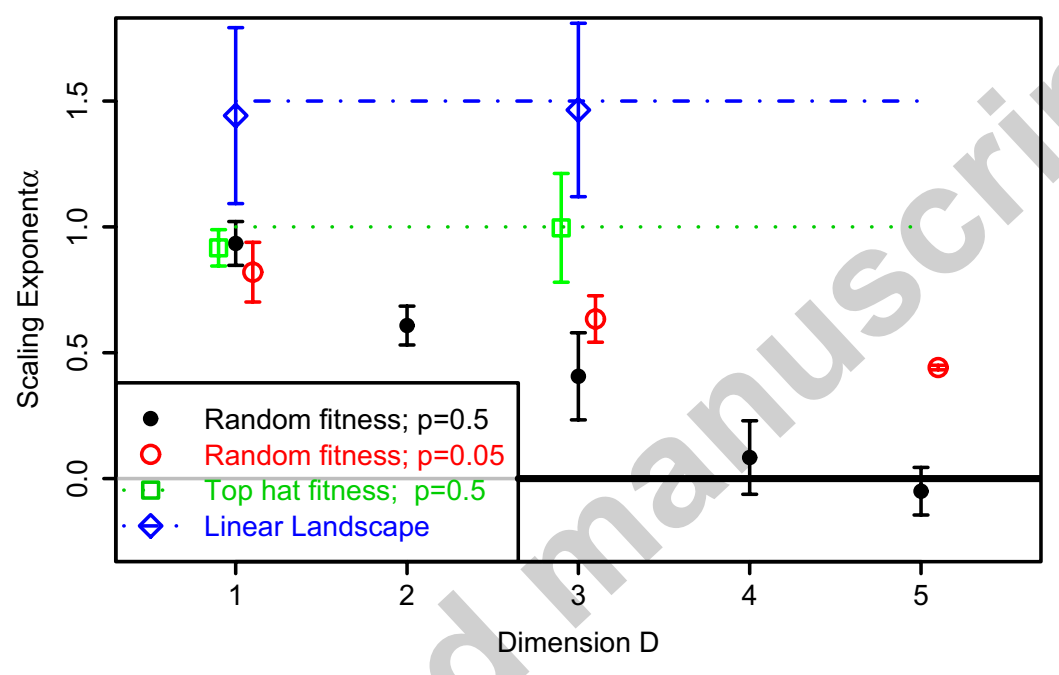

Figure 2: Exponent $\alpha$ for the population size dependence $s_{w}^{*} \propto N^{-\alpha}$ as a function of dimension. Error bars are $95 \%$ confidence intervals for the regression fit for $\alpha$ (linear regression on a $\log$ - $\log$ scale for selection $s$ against population $N)$. Shown are the data the for random fitness landscape (Eq. 1) at $u=0.5$ and $u=0.05$, the 'top-hat' correlated fitness landscape (Eq. 2), and the linear fitness landscape (Eq. 3). Dashed lines correspond to theoretical values. Horizontal perturbations to the dimension have been made for visibility and do not reflect fractal dimensions. 


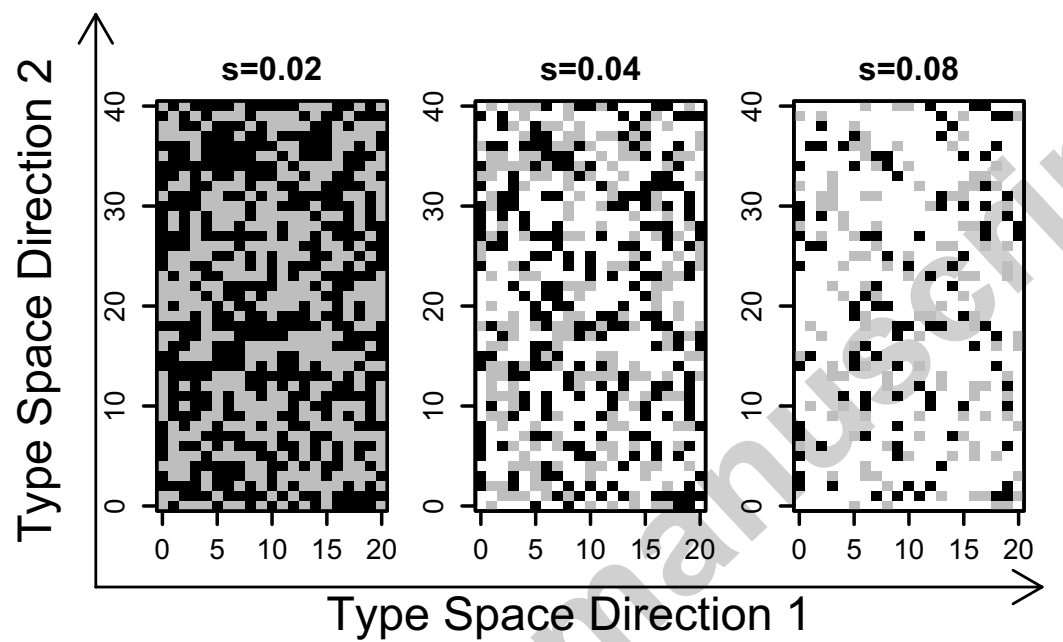

Figure 3: Illustration of 'connectivity' in the random landscape model (Eq. 1) for $D=2$ and $N=100$. The fitness landscape itself is shown. Selection values are (left) $s=0.02$, (middle) $s=0.04$ and (right) $s=0.08$. Types with fitness in the range $(0.99,1)$ compete truly neutrally and are shown in black. Types in grey have fitness in the range $(0.98,0.99)$ which is high enough to survive by chance for moderate times at low population levels. The low mutation rate neutral regime (left) is characterised by a connected network of neutrally competing types (coloured black). However, the neutral regime considered in this paper (middle) allows linking of neutrally competing types by slightly less fit types (coloured grey). At higher selection, connectivity breaks down into isolated clusters and non-neutral dynamics are observed (right). 


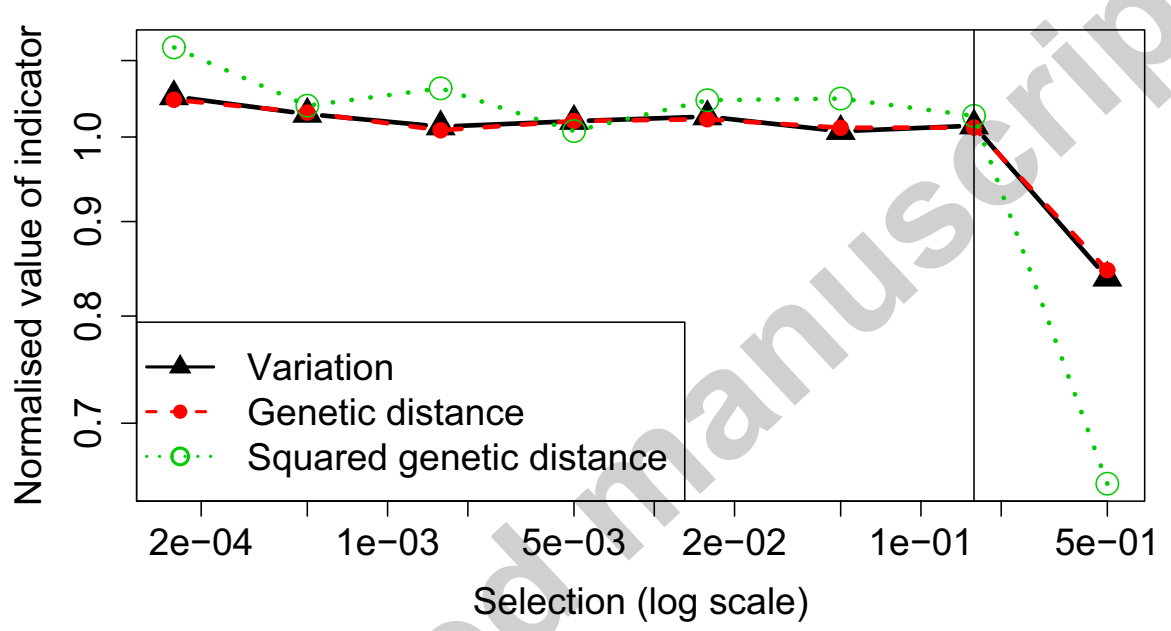

Figure 4: Comparison of 3 different time-averaged neutral indicators for $D=3$ and $N=100$, showing (solid line) the variation $\langle w\rangle$, (dashed line) the mean genetic distance in the population $\langle d\rangle$, and (dotted line) the means square genetic distance $\left\langle d^{2}\right\rangle$, all as a function of selection strength. The critical selection strength $s^{*}$ is identical under all indicators and is shown as a vertical line. 